Ressources en ligne

\title{
L'enseignement de la musique : initiatives et perspectives
}

Federica Minichiello

\section{OpenEdition}

Journals

Édition électronique

URL : https://journals.openedition.org/ries/4537

DOI : $10.4000 /$ ries. 4537

ISSN : 2261-4265

Éditeur

France Education international

Édition imprimée

Date de publication : 1 avril 2016

Pagination : 12-15

ISBN : 978-2-85420-610-4

ISSN : $1254-4590$

\section{Référence électronique}

Federica Minichiello, «L'enseignement de la musique : initiatives et perspectives », Revue internationale d'éducation de Sèvres [En ligne], 71 | avril 2016, mis en ligne le 01 avril 2016, consulté le 03 juillet 2021. URL : http://journals.openedition.org/ries/4537 ; DOI : https://doi.org/10.4000/ries.4537

Ce document a été généré automatiquement le 3 juillet 2021.

(c) Tous droits réservés 


\title{
L'enseignement de la musique : initiatives et perspectives
}

\author{
Federica Minichiello
}

1 L'importance de l'enseignement des disciplines artistiques et culturelles a été affirmée à plusieurs occasions : la conférence internationale de Lisbonne en 2006, l'agenda de Séoul (2010) pour garantir l'accès à un enseignement artistique de qualité et ériger l'art face aux défis sociétaux de notre époque. Le cadre européen de stratégie «Éducation et formation $2020 »$ a également inclus, dans son objectif de créativité et d'innovation, la promotion d'une «sensibilité culturelle».

2 Parallèlement, de nombreux travaux de recherche ${ }^{1}$ se sont penchés sur la relation entre apprentissage artistique et performances cognitives. En 2008, un symposium international de recherche, à l'initiative des ministères français de la culture et de l'éducation, a conclu que peu d'autres disciplines agissent positivement sur une si large palette de compétences ; toutefois, la pérennité de ces effets cognitifs dépendrait de la durée de l'enseignement reçu.

Dans cet article, nous présentons une sélection d'institutions, de politiques et de pratiques éducatives dans un domaine artistique précis: la musique. Nous avons volontairement choisi un périmètre plus étendu que la salle de classe, en raison des synergies possibles entre enseignement formel et non formel.

4 Nous terminons sur l'impact des technologies dans le domaine musical. Il existe un choix important de logiciels, d'applications et d'activités en ligne qui favorisent aujourd'hui la pratique musicale. Les dernières ressources présentées témoignent des perspectives qui s'ouvrent, par la technologie, en termes d'accès, d'interaction et d'apprentissage.

5 Sitographie arrêtée le 10 février 2016. 


\section{Institutions}

\section{International society for music education (ISME)}

6 L'ISME a été fondé lors d'une conférence de l'UNESCO en 1953, avec la mission de promouvoir l'éducation musicale au sein de l'enseignement général. L'institution s'est progressivement transformée en réseau mondial d'éducateurs, réunis à l'occasion d'un séminaire annuel autour des pratiques musicales et des politiques de promotion. La 32e édition aura lieu en juillet 2016 à Glasgow et porte, entre autres, sur la musique communautaire et l'enseignement de la musique pour la petite enfance.

[https://twitter.com/official_isme]; [http://www.isme2016glasgow.org/]

\section{International Music Council (IMC)}

7 L'International Music Council, fondé en 1949 par l'Unesco, est le plus important réseau d'organisations œuvrant dans le domaine, dans environ 15 pays. L'institution publie une lettre d'information mensuelle sur l'actualité de l'éducation musicale, les politiques sectorielles et les travaux de recherche récents. Parmi ses initiatives, on peut citer le programme "African Music Development Programme», pour promouvoir le secteur musical dans des pays africains et le projet Erasmus +: "Music and Resilience Support " (MARS) sur les liens entre psychologie sociale et musique et la formation à distance d'éducateurs spécialisés.

[http://www.imc-cim.org/]

\section{European music council (EMC)}

8 Lié à l'International Music Council (voir supra), ce conseil régional pour l'Europe, créé en 1972, mène un travail d'influence auprès des décideurs politiques pour la promotion de la musique. Parmi ses initiatives, on peut citer l'action en faveur de la reconnaissance des qualifications des musiciens («Bologna and Music») et la préparation du séminaire de Bonn en 2011, qui a traduit les objectifs de l'agenda de Séoul pour la musique. Parmi les recommandations de Bonn : encourager l'accès et la participation à une activité musicale, promouvoir l'emploi de personnel spécialisé dès les premières années d'école, former les musiciens à la pédagogie et les enseignants à la musique.

[http://www.emc-imc.org]

\section{Politiques et initiatives}

\section{"L'éducation artistique et culturelle à l'école en Europe »}

Cette publication d'Eurydice de 2009 porte sur l'enseignement des disciplines artistiques et culturelles, dont la musique, dans l'enseignement primaire et secondaire inférieur de trente pays. Le panorama est hétérogène, en termes d'articulation des programmes ou d'éventail d'activités proposées - la musique fait partie des disciplines régulièrement enseignées. Dans la moitié des pays, les heures d'enseignement varient entre cinquante et cent heures dans le primaire et tendent à se réduire dans le 
secondaire. L'enseignement est confié à des éducateurs généralistes dans le primaire, à l'exception du Danemark, de l'Allemagne et de la Lettonie, qui optent pour un personnel spécialisé à tout niveau d'enseignement.

[http://eacea.ec.europa.eu/education/eurydice]

\section{Le Royaume-Uni}

Le Royaume-Uni s'est doté en 2012 d'un plan national pour l'éducation musicale et de pôles sectoriels dédiés (« musical hubs») pour offrir à chaque enfant âgé de 5 à 18 ans la possibilité d'apprendre un instrument - sur une durée préconisée d'un an. Le plan prévoit des mesures comme la mise en place d'une qualification d'éducateur musical, afin de professionnaliser le métier; des prêts à taux zéro pour faciliter l'achat d'instruments; des modules de formation destinés aux enseignants. L'évaluation du programme en 2014 semble positive : les pôles travaillent avec presque 84 \% des écoles publiques, favorisent la création d'orchestres d'élèves (surtout pour les enfants de 7 à 11 ans) et les trois quarts des élèves concernés ont acquis un niveau débutant, bien que le plan soit trop récent pour connaitre l'évolution dans le temps de ces compétences. [http://www.artscouncil.org.uk/]

\section{La France}

11 L'éducation musicale figure dans les programmes scolaires français de la maternelle au collège et se poursuit au lycée sur une base facultative ; l'accent est particulièrement mis sur la pratique du chant, avec le lancement, en février 2015, de l'opération « l'école en chœur ", une sélection académique et nationale des meilleurs vidéos de chorales scolaires. Parmi les actions qui favorisent une pratique instrumentale, on peut évoquer les classes à horaires aménagés musique, dites "cham», qui permettent un aménagement de l'emploi du temps des élèves afin d'inclure des cours de musique dans un conservatoire ${ }^{2}$ et l'initiative "Orchestres à l'école", lancée en 2000 par la chambre syndicale de la facture instrumentale. Selon une évaluation de l'Institut Montaigne en 2011, la pratique orchestrale (pendant trois ans) se traduirait par une amélioration de la moyenne générale, des notes de vie scolaire et une meilleure attitude des élèves vis-à-vis de l'école.

[http://eduscol.education.fr/] ; [http://www.orchestre-ecole.com/]

\section{La Confédération suisse}

En 2012, plus de $72 \%$ des Suisses ont voté par référendum en faveur de l'inscription de la formation musicale dans leur constitution, pour défendre un accès plus égalitaire à la pratique de la musique. Le programme quinquennal pour la culture 2016-2020 prévoit un financement à hauteur d'un milliard de francs suisses; parmi les mesures en musique : le soutien de camps musicaux et de cours de musiques pour les 6-20 ans, la formation d'une centaine de moniteurs agréés et une revue à la baisse des tarifs des écoles de musique pour les élèves et les apprentis.

[http://www.bak.admin.ch] 


\section{Music in Africa}

13 Le portail «Music In Africa » est géré par la fondation homonyme, fondée en 2013 en partenariat avec l'Institut Goethe (https://www.goethe.de) et la Fondation Siemens (http://www.siemens-fondation.fr/). Ce site propose de nombreuses ressources sur la pratique de la musique dans les pays africains, en particulier sur les différentes politiques culturelles et éducatives : on y découvre qu'à Madagascar, la musique est généralement une activité parascolaire en option dans les écoles d'expressions françaises $^{3}$; qu'en Côte d'Ivoire, elle figure dans les programmes officiels mais subit une pénurie d'enseignants spécialisés et une influence excessive de la culture occidentale - par exemple, l'enseignement de la théorie musicale est maintenu, malgré une tradition locale principalement orale.

[http://musicinafrica.net/]

\section{El Sistema}

14 El Sistema est un système national d'orchestres et de chœurs créé en 1975 au Venezuela, s'adressant en priorité aux enfants des quartiers défavorisés des grandes villes; fortement appuyé par le gouvernement vénézuélien, ce programme a été introduit dans les cursus des écoles publiques et a touché plus de 500000 enfants. Le modèle est actuellement reproduit dans plus de 90 pays.

15 Les détracteurs dénoncent un effet médiatique qui oublierait les plus démunis, des relations controversées avec le gouvernement, le manque de débouché pour les musiciens et la faible recherche dans les effets sociaux du programme. La banque interaméricaine de développement a cependant réalisé une évaluation d'impact en 2012, qui démontre une baisse liée du taux de décrochage scolaire et de criminalité.

[http://fundamusical.org.ve]

\section{Technologie et perspectives}

\section{Orchestre interactif}

16 «Online Orchestra» est un projet de recherche mené par l'Université de Falmouth (Grande-Bretagne), qui explore les possibilités en termes de connectivité pour rendre la pratique orchestrale plus accessible à des enfants et des musiciens amateurs éloignés, en les amenant à jouer ensemble « à distance ». Une première performance a eu lieu en juillet 2015. La même approche est également étudiée au Danemark : la Philharmonie de Copenhague proposera en mai 2016 une expérience orchestrale en interaction et cocréation avec le public; un projet d'orchestre interactive, World Online Orchestra, semble également à l'étude (une campagne de financement participatif a eu lieu en 2015).

[http://onlineorchestra.com/] ; [http://www.copenhagenphil.dk/]

\section{Interaction son / musique / mouvement}

17 Musique Tech Fest est un temps de création qui réunit inventeurs, artistes, chercheurs et industriels autour des innovations technologiques, pour explorer des moyens inédits 
de jouer avec la technologie, le corps, la voix et donner vie à des instruments et des expériences musicalement novateurs. Dans le même domaine, on peut également citer le travail de l'institut de recherche français IRCAM: partitions interactives, instruments augmentés, le projet d'interaction musicale Rapid-Mix dans le cadre du programme Horizon 2020, etc. ${ }^{4}$

[http://musictechfest.net]; [http://www.ircam.fr/]

\section{GeorgiaTech Center for Music Technology}

Ce centre de recherche étudie le déploiement de technologies pour transformer l'expérience musicale. L'unité robotique, par exemple, expérimente les interactions musicales, l'improvisation et la collaboration en temps réel entre hommes et machines. Le programme EarSketch, à destination d'étudiants de lycée et d'université, explore l'utilisation pédagogique de la musique pour développer des compétences en informatique par la composition et "la sonification", la transformation de jeux de données en musique (le nombre de mots dans un poème, les cotations boursières, etc.). [http://gtcmt.gatech.edu]

\section{NOTES}

1. La Documentation française, "Évaluer les effets de l'éducation artistique et culturelle ", symposium européen et international de recherche, Centre Pompidou (2008) et OCDE, «L'art pour l'art? L'impact de l'éducation artistique » (2014).

2. La base de données de la Cité de la musique recense 152 conservatoires impliqués dans un projet de classe « cham » : http://gpm.cite-musique.fr.

3. Écoles qui proposent à leurs élèves un enseignement basé sur le programme scolaire français (et les examens officiels).

4. Instruments augmentés : « instruments acoustiques auxquels sont intégrés des capteurs, afin de transmettre en temps réel des paramètres gestuels »; projet Rapid-Mix : projet européen dont l'objectif est de «développer de nouveaux modes d'interaction musicale en intégrant des données multimodales liées aux mouvements et au corps» (source : IRCAM).

\section{INDEX}

Mots-clés : éducation artistique, éducation musicale

Keywords : arts education, music education

Palabras claves : educación artística, educación musical 


\section{AUTEUR}

\section{FEDERICA MINICHIELLO}

Federica Minichiello est chargée de veille au centre de ressources et d'ingénierie documentaires (CRID) du Centre international d'études pédagogiques (CIEP). Courriel : minichiello@ciep.fr 